\title{
Virtual Home Assistants for People with Dysarthria
}

\author{
Aisha Jaddoh \\ Cardiff University \\ School of Computer Science and Informatics \\ Cardiff, UK \\ jaddoha@cardiff.ac.uk
}

\begin{abstract}
Many individuals with disabilities use virtual home assistants (VHAs) to communicate and execute daily tasks (Pradhan, Mehta \& Findlater 2018). Because VHAs are controlled by verbal communication, some groups of users, including people with speech impairments, face barriers to effective interaction with VHAs. My research addresses the problems that individuals with speech impairments, specifically those with dysarthria, experience in interacting with these devices. I propose an alternate technique for interaction using nonverbal voice cues, such as humming, hissing and vowel sounds. The technique requires from the user only the ability to vocalise.
\end{abstract}

Accessibility, Virtual home assistants, Nonverbal interaction, Speech impairment, Dysarthria, Digital home assistant

\section{INTRODUCTION}

This study in the areas of accessibility and assistive technology focuses on speech impairment disability and dysarthria. I aim to give people with dysarthria a voice and to enable them to use technology as do their peers without such disability by proposing a more easily usable, accessible solution for their use. In this regard, extensive research has been conducted on speech impairment disability, but mainly from the artificial intelligence and machine learning perspectives. The studies based on machine learning consider ways to improve speech recognition, whereas those on human-computer interaction investigate this issue from a different perspective. Since 2016, when the first virtual home assistant (VHA) was released, people have relied on them. Various companies have produced VHAs, including Google Assistant (Google n.d.), Amazon Echo (Black 2019) and Apple HomePod (Apple n.d.). Users control these devices through speech commands to implement some action, such as increasing or decreasing the room temperature or playing a favourite song. The technology behind the device is automatic speech recognition, in which speech is received, processed and then transcribed automatically (Young \& Mihailidis 2010). However, people with dysarthria may face problems in using VHAs. Dysarthria, a type of speech impairment associated with a neurological condition characterised by weakness in the face, lip, tongue and throat muscles, is caused by brain damage from a stroke, brain injury, amyotrophic lateral sclerosis or other condition. Hence, in this study, I propose an alternate solution involving nonverbal commands for interacting with VHAs.

\section{RELATED LITERATURE}

Some studies have shed light on the challenges that individuals with dysarthria face when interacting with VHAs and voice assistants in general. A key issue is inaccurate speech recognition when the device transcribes received speech incorrectly, resulting in a high word error rate (WER). Further, studies have reported that when dysarthria severity increases, the WER also increases (Mustafa, M.B., Rosdi, F., Salim, S.S. and Mughal, M.U 2015). Ballati, Corno and De Russis (2018a) studied the interactions of users with dysarthria with three voice assistants: Siri, Google Assistant and Amazon Echo and showed that the recognition percentage was $50 \%$ to $60 \%$. Adopting a similar approach, De Russis and Corno (2019) investigated the interactions with IBM Watson Speech to Text, Google Cloud Speech and Microsoft Azure of speakers with dysarthria and found that the WER reached $80 \%$ to $90 \%$. Other studies have considered research areas such as the communication and speech of individuals with dysarthria. Ansel and Kent (1992) revealed that this impairment leads to speech unintelligibility and difficulty in articulating. The more severe the case, the greater the unintelligibility. In addition, these individuals experience breathing problems while speaking, which makes speech even more difficult 
for them. Moreover, the fatigue this impairment causes makes it difficult for them to vocalise long sentences. Thus, these findings in the literature support the use of nonverbal interaction as a communication method because it will overcome the aforementioned challenges.

\section{PROPOSED SYSTEM}

Because dysarthria may deteriorate over time (Kent et al. 1999), reaching a level at which speakers cannot produce intelligible speech (Ballati, Corno \& De Russis 2018b), and in light of the current knowledge about the performance of VHAs and voice assistants when used by these speakers, I propose a system that allows people with dysarthria to interact with VHAs using nonverbal cues. The system consists of a microphone connected to a Raspberry Pi computer that receives the voice cue from the speaker. The Raspberry Pi translates the voice cue using machine learning and classification models, and sends the translated command to the VHA iCloud service, which performs the action requested by the user by sending the reply back to either a VHA or any home Internet of Things device. The nonverbal voice cue is any sound that the individual produces. It could be a hiss, a vowel, a pitch-based tone (such as humming) or a combination of these. The system is written in Python and will be tested using nonverbal recordings from the TORGO database. This database is a repository of recordings by speakers with dysarthria for research purposes.

\section{REFERENCES}

Ansel, BM \& Kent, RD 1992, 'Acoustic-phonetic contrasts and intelligibility in the dysarthria associated with mixed cerebral palsy', Journal of Speech, Language, and Hearing Research, vol. 35, no. 2, pp. 296-308.

Apple n.d., The new sound of home, viewed 21 May 2020 <https://www.apple.com/uk/homepod/>.
Ballati, F, Corno, F \& De Russis, L 2018a, 'Hey Siri, do you understand me?' Virtual assistants and dysarthria', Intelligent Environments (Workshops), pp. 557-66.

Ballati, F, Corno, F \& De Russis, L 2018b, 'Assessing virtual assistant capabilities with Italian dysarthric speech', Proceedings of the 20th International ACM SIGACCESS Conference on Computers and Accessibility, pp. 93-101.

Black, M 2019, What is Amazon Echo? A complete guide, viewed 21 May 2020, <https://www.techadvisor.co.uk/news/audio/ama zonecho-3584881/>.

De Russis, L \& Corno, F 2019, 'On the impact of dysarthric speech on contemporary ASR cloud platforms', Journal of Reliable Intelligent Environments, vol. 5, no. 3, pp. 163-72.

Google n.d., Meet Google Home, viewed 21 May 2020, https://store.google.com/gb/product/google_hom e_speaker>.

Kent, RD, Weismer, G, Kent, JF, Vorperian, HK \& Duffy, JR 1999, 'Acoustic studies of dysarthric speech: methods, progress, and potential', Journal of Communication Disorders, vol. 32, no. 3, pp. 141-86.

Mustafa, M.B., Rosdi, F., Salim, S.S. and Mughal, M.U., 2015. Exploring the influence of general and specific factors on the recognition accuracy of an ASR system for dysarthric speaker. Expert Systems with Applications, 42(8), pp.3924-3932.

Pradhan, A, Mehta, K \& Findlater, L 2018, 'Accessibility came by accident': use of voicecontrolled intelligent personal assistants by people with disabilities', Proceedings of the 2018 $\mathrm{CHI}$ Conference on Human Factors in Computing Systems, pp. 1-13.

Young, V \& Mihailidis, A 2010, 'Difficulties in automatic speech recognition of dysarthric speakers and implications for speech-based applications used by the elderly: a literature review', Assistive Technology, vol. 22 no. 2, pp. 99-112. 